\title{
A Novel Transabdominal Preperitoneal Hernioplasty Technique in the Repair of Large Inguinal Hernias: The use of Polypropylene Mesh in the form of Plug-patch
}

\author{
Hasan Calis, Serkan Sengul, Yilmaz Guler and Zulfikar Karabulut \\ Department of General Surgery, Alanya Alaaddin Keykubat University Training and Research Hospital, Alanya, Turkey
}

\begin{abstract}
Objective: To describe the outcome of modified transabdominal laparoscopic preperitoneal (TAPP) hernioplasty repair with plug-patch of polypropylene mesh.

Study Design: A descriptive study.

Place and Duration of Study: Alanya Alaaddin Keykubat University Training and Research Hospital, Alanya, Turkey from December 2017 to December 2019.

Methodology: Patients aged 18-70 years, who underwent TAPP hernioplasty at this clinic between December 2017 and 2019 were evaluated retrospectively. Those with a defect diameter of $3 \mathrm{~cm}$ and above, which were measured intraoperatively, were considered as large inguinal hernias. A modified TAPP procedure that includes the plug-patch technique, using polyester mesh, similar to that of open inguinal hernia repair, was performed on these patients. Complications and recurrences frequency was noted.

Results: This modified TAPP procedure was applied to 35 patients (24 men, 11 women). The defect size was 3-4 cm in 24 patients, and $4 \mathrm{~cm}$ in 11 patients. None of the patients developed chronic pain. Five patients had a feeling of fullness in the inguinal region where the repair was performed, and their complaints subsided at the first month of follow-up. Seroma developed in 2 patients, but resolved spontaneously. During the follow-up period, no chronic complications or recurrences were observed.

Conclusion: This technique is simple and does not create a serious cost burden on TAPP technique. Furthermore, it can be applied routinely with the advantages of low pain, rapid recovery and acceptable complication rates as well as the reduction of pseudo-recurrence rates.
\end{abstract}

Key Words: Hernia, Laparoscopic repair, Plug-patch technique, Modified TAPP, Mesh.

How to cite this article: Calis H, Sengul S, Guler Y, Karabulut Z. A Novel Transabdominal Preperitoneal Hernioplasty Technique in the Repair of Large Inguinal Hernias: The use of Polypropylene Mesh in the form of Plug-patch. J Coll Physicians Surg Pak 2021; 31(07):825-828.

\section{INTRODUCTION}

Several techniques have been described in inguinal hernia repair to minimise complications and improve outcomes. The main aim of the treatment is to improve the recurrence rates and diminish the chronic pain that impairs the comfort of life. ${ }^{1}$ However, it is really difficult to choose the best operation technique for the patient. Because this decision also depends on numerous factors such as the characteristics of the hernia, the type of anesthesia, the preference of the surgeon and the patient, the training and capability of the surgeon, geographic differences and equipment.

Correspondence to: Dr. Hasan Calis, Department of General Surgery, Alanya Alaaddin Keykubat University Training and Research Hospital, Alanya, Turkey

E-mail: drhasancalis@hotmail.com

Received: August 12, 2020; Revised: October 18, 2020;

Accepted: December 10, 2020

DOI: https://doi.org/10.29271/jcpsp.2021.07.825
Many different techniques of laparoscopic inguinal hernia repair have been defined and modified. Among these, transabdominal laparoscopic preperitoneal (TAPP) repair is one of the most preferred techniques. Modifications of this technique have also been described over time. The main purpose of TAPP surgeries for inguinal hernia is to close both direct and indirect areas with mesh after dissection. ${ }^{2-4}$ However, there is no defined technique for the direct repair or closure of the defect within these modifications. Specially in large inguinal hernias, as a result of protrusion of the mesh into the defect, pseudo-recurrence may occur due to the bulging of the mesh from the defect. Therefore, in TAPP repair, the authors developed a new technique which modified the classical TAPP technique by using polyester mesh in a similar manner with the plug-patch technique applied in the classical open method before placing the mesh on the dissected field, and named this technique as 'Calistechnique'. 
The aim of this study was to determine the effectiveness of this technique in terms of complications and recurrence.

\section{METHODOLOGY}

Patients aged between 18 and 70 years, who underwent TAPP at General Surgery Clinic, Alanya Alaaddin Keykubat University Training and Research Hospital, Alanya, Turkey between December 2017 and December 2019 were evaluated retrospectively. Those with a defect diameter of $3 \mathrm{~cm}$ and above, which were measured intraoperatively, were considered as large inguinal hernias. ${ }^{5}$ 'Calis technique', a modified TAPP procedure that includes the plug-patch technique, using polyester mesh, similar to that of open inguinal hernia repair, was performed on these patients. The data regarding peroperative period of these patients were examined. Inguinal hernia was categorised indirectly and directly, depending on the location. Written informed consents were obtained from all patients prior to the procedure. Patients who could not be reached in the routine follow-up protocol after the procedure, were excluded from the study.

The classical TAPP herniorrhaphy technique was applied to all patients under general anesthesia; and in the Trendelenburg position with arms and legs closed, with three port entries. In the laparoscopic system, $10 \mathrm{~mm}$ camera and $5.0 \mathrm{~mm}$ instruments were used. A $10 \mathrm{~mm}$ incision was made just above the umbilicus and a $10 \mathrm{~mm}$ port was forwarded into the abdomen by the open method. Then pneumoperitoneum was created with carbon dioxide at 8-12 $\mathrm{mmHg}$ pressure. Two $5 \mathrm{~mm}$ ports were placed bilaterally in the middle clavicular line $1 \mathrm{~cm}$ below the umbilical line using $30 \mathrm{~mm}$ camera. After the epigastric vessels, spermatic vessels, vas deferens or round ligaments, urachus, iliac vessels, and bladder were defined anatomically, peritoneum was incised transversely toward medial line passing over the point where the anterior superior iliac spine intersects the hernia defect. The dissection was performed up to the retroinguinal spaces and the preperitoneal area was enlarged. Meanwhile, Cooper's and Gimbernat's ligaments were exposed; corona mortis and external iliac vessels were preserved. The hernia sac was carefully dissected. If the defect was $3 \mathrm{~cm}$ or above, $7 \times 7 \mathrm{~cm}$ polyester mesh has been turned into an inverted cone shape by placing 2 sutures with 2/0 prolene in the extra-abdominal area. Afterwards, it was sent into the abdomen through the $10 \mathrm{~mm}$ port and was placed into the defect as if it were a plug (Figure 1). This plug was fixed to the surrounding tissues by tucker (Figure 2). Then, $10 \times 15 \mathrm{~cm}$ polyester mesh (second layer) was placed on top of the defect, which was closed like a plug with a mesh, and fixed with a tucker (Figure 3). After the peritoneum was closed with the help of tucker, trocars were removed under the visual control of a camera. The umbilical $10 \mathrm{~mm}$ port defect was closed followed by closure of skin incisions in all ports.

In the postoperative period, a single dose of dexketoprofen was given to patients and in need of more analgesic were received a second dose. Pain assessments were done with visual analog scale on the first postoperative day. All patients were discharged with a prescription of oral dexketoprofen on the postoperative first day. First week, month and second month follow-ups were done and the patients received physical examination on each follow-up visit. Those who completed 6 months and one year in their follow-up, were called again for control. All patients were recommended to contact the authors for re-examination in cases such as delayed complications or unexpected problems, especially chronic pain (pain lasting more than 3 months) orswelling.

Descriptive statistics were analysed with Microsoft Excel 2016 by recording the demographic data of the patients as well as the diagnosis-treatment and postoperative period data. Qualitative data were expressed as numbers and percentages, while quantitative as mean \pm S.D.

\section{Table I: Patients' data.}

\begin{tabular}{|c|c|}
\hline Age (years) & $37.8 \pm 14.2$ \\
\hline \multicolumn{2}{|l|}{ Gender } \\
\hline Male & $24(68.6 \%)$ \\
\hline Female & $11(31.4 \%)$ \\
\hline BMI $\left(\mathrm{kg} / \mathrm{m}^{2}\right)$ & $24.1 \pm 3.8$ \\
\hline \multicolumn{2}{|l|}{ Symptom } \\
\hline Right & $17(48.6 \%)$ \\
\hline Left & $10(28.6 \%)$ \\
\hline Both & $8(22.8 \%)$ \\
\hline Direct hernia & $27(77.1 \%)$ \\
\hline Indirect hernia & $16(45.7 \%)$ \\
\hline Before hernia surgery & $7(20 \%)$ \\
\hline \multicolumn{2}{|l|}{ Defect size } \\
\hline$>3,<4 \mathrm{~cm}$ & $24(68.6 \%)$ \\
\hline $4 \mathrm{~cm}$ & $11(31.4 \%)$ \\
\hline \multicolumn{2}{|l|}{ Visual analog scale } \\
\hline Postop 1 day & $2.9 \pm 1.1$ \\
\hline Postop 1 week & $1.8 \pm 0.9$ \\
\hline Postop 1 month & $0.4 \pm 0.6$ \\
\hline Mean operation time (second) & $46.4 \pm 8.8$ \\
\hline Seroma & $2(5.7 \%)$ \\
\hline Chronic pain & $0(0 \%)$ \\
\hline Prolonged hospitalisation & $1(2.9 \%)$ \\
\hline Return daily life (postoperative day) & $3.6 \pm 1.8$ \\
\hline Recurrence & $0(0 \%)$ \\
\hline Follow-up period (month) & $21 \pm 5.1$ \\
\hline
\end{tabular}

This modified TAPP procedure which was defined, was applied to 36 patients. A total of 35 patients [ 24 (68.6\%) men, and 11 $(31.4 \%)$ women] were included in the study since one patient could not be reached during their follow-up. Eight $(22.8 \%)$ of these patients underwent bilateral modified TAPP repair. The results were shown in Table I.

On the first postoperative day, all patients had only mild pain (mean visual analog scale $<3$ ). None of the patients developed chronic pain. Five (14.3\%) patients had a feeling of fullness in the inguinal region where the repair was performed, and their complaints subsided at the first month follow up. Seroma developed in 2 (5.7\%) patients, but resolved spontaneously. Only one $(2.9 \%)$ patient was hospitalised for 48 hours, longer due to anaphylaxis developing after analgesic (dexketoprofen) 
administration. During the follow-up period, no chronic complications or recurrences were observed.

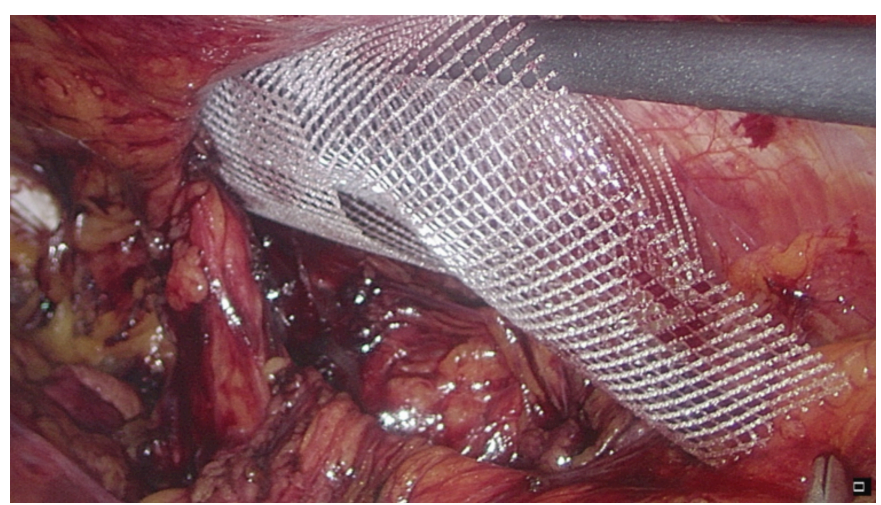

Figure 1: Insertion of the plug mesh.

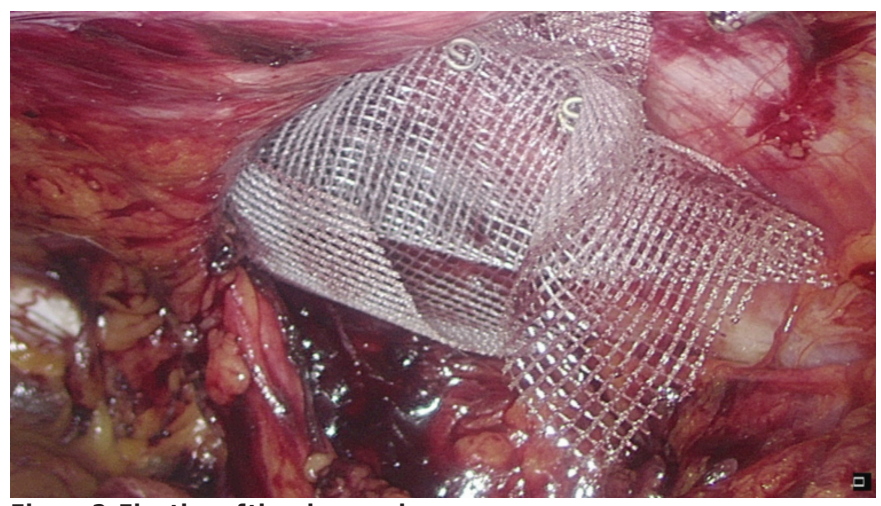

Figure 2: Fixation of the plug mesh.

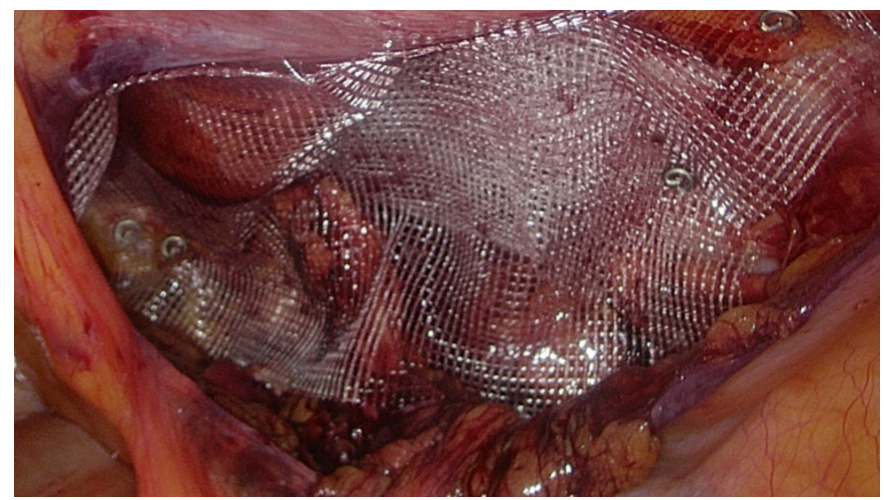

Figure 3: Fixation of the second layer mesh.

\section{DISCUSSION}

Surgical repair of inguinal hernias, both open and laparoscopic, is one of the most commonly performed general surgery procedures worldwide.$^{6,7}$ Laparoscopic inguinal hernia repair has the advantage of less postoperative pain and early return to work compared to open repair. ${ }^{2}$ TAPP, a commonly applied procedure, is one of the laparoscopic hernia repair methods that use meshes to close the hernia defect. The biggest advantage of TAPP is that it enables to categorise the type of hernia correctly by visualising the defect and anatomical structures intra-abdominally. ${ }^{3,4}$

Although TAPP is widely accepted with its various advantages, relapse rates have been reported up to $6 \%$ and the most common complications include hematoma, seroma, pain and bulging of the mesh. ${ }^{8,9}$ In TAPP repair, a wide peritoneal dissection is required for the placement of the mesh without tension in order to cover the defect widely. In addition, since the defect is not closed, hernias with large defects may also manifest complications that may develop after the procedure such as mesh bulging from the defect and chronic pain. ${ }^{8}$ Specially in large inguinal hernias, as a result of protrusion of the mesh into the defect, pseudo-recurrence may occur due to the bulging of the mesh from the defect. ${ }^{2}$

There is no clear information on the repair or direct closure of the defect in laparoscopic inguinal hernia surgeries in the current guidelines. When the defect is large, the risk of recurrence is relatively higher. ${ }^{10,11}$ In fact, retraction of the mesh and closure of the defect with small mesh are considered as one of the main mechanisms of recurrence after laparoscopic inguinal hernia repair. ${ }^{12}$ The risk of recurrence can be doubled in small mesh use. ${ }^{2,8,13}$ In support of this, the general view regarding the use of mesh in hernia repair is that the mesh should be placed so that it exceeds the defect at least 3 or $4 \mathrm{~cm}$ in all directions. Accordingly, it is believed that recurrence will decrease with the use of large mesh. However, specially in hernias with a large defect $(>3 \mathrm{~cm})$, even the use of large mesh and fixing the mesh may be insufficient, thus the protrusion of the mesh into the defect occurs and pseudo-recurrences may develop few months later. ${ }^{2,14,15}$ In fact, the problem here is not that the mesh does not exceed the defect by $3 \mathrm{~cm}$, but the defect is not closed at all. Therefore, in TAPP repair, the authors developed the Calis technique, which was modified by the classical TAPP technique by using polyester mesh in a similar way to the plug-patch technique applied in the classical open method before placing the mesh on the dissected field. This technique is simple, inexpensive, and prevents both pseudo-recurrences and recurrences that may occur in the early period. While the size of the defect measured intraoperatively was $3-4 \mathrm{~cm}$ in 24 patients, it was $4 \mathrm{~cm}$ in 11 patients. In most inguinal hernia repairs performed using mesh, recurrence occurs within 2 years after surgery. ${ }^{8}$ No recurrence was observed in our patient group for an average of 21 months.

This modified TAPP technique, which was described, is a method that can be done by any surgeon who is able to perform an ordinary laparoscopic inguinal hernia repair, and it is simple enough not to require special skills. The crucial point here is that tucker fixes the graft that is plugged into the tissue. Otherwise, migration of the plug graft into the defect will be inevitable during the postoperative period. No complication was observed in any patient in this study. None of the patients developed chronic pain.

The most important question regarding this technique was its close association with spermatic cord and gonadal vascular structures, when applied in indirect hernias. However, the authors thought that the graft used in ordinary TAPP technique was in direct contact with these structures, so there would be no problem. No problems developed during the mean follow-up period of 21 months. 
Many authors reported approximately $5 \%$ rate of seroma development, which is one of the most common minor complications after laparoscopic inguinal hernia repair; and various techniques have been applied to reduce seroma development. ${ }^{8}$ In this study, seroma developed and regressed spontaneously in 2 patients. These seromas did not pose a serious problem in these patients.

This is a retrospective study and since it was performed in a single centre, the number of patients was not very large. The mean follow-up was 21 months and longer follow-up would be required to achieve long-term recurrence rates.

\section{CONCLUSION}

The application of this modified technique is simple and does not create a serious cost burden on TAPP technique. Furthermore, the authors believe that it is a technique that can be applied routinely with the advantages of low pain, rapid recovery and acceptablecomplication ratesaswell as the reduction of pseudo-recurrence rates.

\section{ETHICALAPPROVAL:}

This study was conducted after obtaining approval from the Institutional Review Board (15-8. 09.01.2020).

\section{PATIENTS' CONSENT:}

Informed consents were obtained from all participants included in the study.

\section{CONFLICT OF INTEREST:}

The authors declared no conflict of interest.

\section{AUTHORS' CONTRIBUTION:}

HC: Concept, design, patient files, interpretation, draft.

SS: Drafting work, critical analysis.

YG: Data analysis, SPSS work, patientfiles.

ZK: Final approval, logistics, critical analysis.

\section{REFERENCES}

1. Hernia Surge Group. International guidelines for groin hernia management. Hernia 2018; 22(1):1-165. DOI: 10.1007/s10029-017-1668-x.

2. Li J, Zhang W. Closure of a direct inguinal hernia defect in laparoscopic repair with barbed suture: A simple method to prevent seroma formation? Surg Endosc. 2018; 32(2): 1082-6. DOI: 10.1007/s00464-017-5760-1.

3. Bittner R, Arregui ME, Bisgaard T, Dudai M, Ferzli GS, Fitzgibbons RJ, et al. Guidelines for laparoscopic (TAPP) and endoscopic (TEP) treatment of inguinal hernia [International endohernia society (IEHS)]. Surg Endosc 2011; 25(9): 2773-2843. DOI: 10.1007/s00464-011-1799-6.

4. Bittner R, Montgomery MA, Arregui E, Bansal V, Bingener J, Bisgaard $\mathrm{T}$, et al. Update of guidelines on laparoscopic
(TAPP) and endoscopic (TEP) treatment of inguinal hernia (International Endohernia Society). Surg Endosc 2015; 29(2): 289-321. DOI: 10.1007/s00464-014-3917-8.

5. Miserez M, Alexandre JH, Campanelli G, Corcione F, Cuccurullo D, Pascual MH, et al. The European hernia society groin hernia classification: simple and easy to remember. Hernia 2007; 11(2):113-6. DOI: 10.1007/ s10029-007-0198-3.

6. Bracale U, Andreuccetti J, Sodo M, Merola G, Pignata G. Lack of advantages of slit mesh placement during laparoscopic transabdominal preperitoneal inguinal hernia repair (TAPP): A single centre, case matched study. BMC Surg 2018; 18(1):75. DOI: 10.1186/s12893-018-0409-0.

7. Bracale U, Rovani M, Picardo A, Merola G, Pignata G, Sodo $M$, et al. Beneficial effects of fibrin glue (Quixil) versus Lichtenstein conventional technique in inguinal hernia repair: A randomised clinical trial. Hernia 2014; 18(2): 185-92. DOI: 10.1007/s10029-012-1020-4.

8. Lee SR, Park SS. The novel technique of transabdominal preperitoneal hernioplasty herniorrhaphy for direct inguinal hernia: Suture repair of hernia defect wall. J Laparoendosc Adv Surg Tech A 2018; 28(1):83-8. DOI: 10.1089/lap. 2017.0045.

9. Pokorny $H$, Klingler A, Schmid T, Fortelny R, Hollinsky C, Kawji $\mathrm{R}$, et al. Recurrence and complications after laparoscopic versus open inguinal hernia repair: Results of a prospective randomized multicenter trial. Hernia 2008; 12(4):385-9. DOI: 10.1007/s10029-008-0357-1.

10. Bittner R, Schwarz J. Inguinal hernia repair: Current surgical techniques. Langenbecks Arch Surg 2012; 397(2):271-82. DOI: 10.1007/s00423-011-0875-7.

11. Lau H. Fibrin sealant versus mechanical stapling for mesh fixation during endoscopic extraperitoneal inguinal hernioplasty: A randomised prospective trial. Ann Surg 2005; 242(5):670-5. DOI: 10.1097/01.sla.0000186440. 02977.de.

12. Silvestre AC, de Mathia GB, Fagundes DJ, Medeiros LR, Rosa MI. Shrinkage evaluation of heavyweight and lightweight polypropylene meshes in inguinal hernia repair: $A$ randomised controlled trial. Hernia 2011; 15(6):629-34. DOI: 10.1007/s10029-011-0853-6.

13. Stengel D, Bauwens K, Ekkernkamp A. Recurrence risks in randomised trials of laparoscopic versus open inguinal hernia repair: To pool or not to pool (this is not the question). Langenbecks Arch Surg 2004; 389(6):492-8. DOI: 10.1007/s00423-004-0512-9.

14. Clapp ML, Hicks SC, Awad SS, Liang MK. Trans-cutaneous closure of central defects (TCCD) in laparoscopic ventral hernia repairs (LVHR). World J Surg 2013; 37(1):42-51. DOI: 10.1007/s00268-012-1810-y.

15. Tse GH, Stutchfield BM, Duckworth AD, de Beaux AC, Tulloh B. Pseudorecurrence following laparoscopic ventral and incisional hernia repair. Hernia 2010; 14(6):583-7. DOI: 10.1007/s10029-010-0709-5. 